\title{
Effect of Level of Presentation to Listeners on Scaled Speech Intelligibility of Speakers with Dysarthria
}

\author{
Yunjung Kim ${ }^{a}$ Christina Kuo ${ }^{\text {b }}$ \\ a Department of Communication Sciences and Disorders, Louisiana State University, Baton Rouge, La., and \\ ${ }^{b}$ Department of Communicative Disorders, Waisman Center, University of Wisconsin-Madison, Madison, Wisc., USA
}

\section{Key Words}

Presentation level · Speech intelligibility · Dysarthria

\begin{abstract}
Objective: This study examined the effect of intensity level of presentation on scaling of speech intelligibility in speakers with and without dysarthria. Patients and Methods: A total of 50 utterances produced by speakers with dysarthria and healthy speakers were played to 60 listeners in four conditions, which consisted of two different presentation levels ('high' vs. 'low') and equalization of levels across utterances ('adjusted' vs. 'unadjusted'). Speech intelligibility was scaled by using a direct magnitude estimation technique with and without modulus. Results: A significant decrease in speech intelligibility was indicated when the stimuli were adjusted to have fixed intensity on the most intense vocalic nuclei of each word, while no significant change was found between 'high' and 'low' presentation level conditions. Conclusion: The findings suggest that an increase in presentation level alone does not result in significant improvement in speech intelligibility ratings. The results are discussed by considering clinical implications in conducting speech therapy with emphasis on intensity variation.
\end{abstract}

Copyright $\odot 2011$ S. Karger AG, Basel

\section{Introduction}

Speaking (voice) intensity has been noted as one of the key factors contributing to audibility of various acoustic cues in the speech perception process. Presumably, any effect of speech intensity on acoustic cues would also affect speech intelligibility of naturally produced utterances. For example, the percentage of correct word identification or segment discrimination has been reported to vary as a function of signal-to-noise ratio, whether the noise is defined as quiet or some form of constant noise $[1,2]$.

The relationship between signal level and perceptual aspects of speech has been of particular interest in speakers with dysarthria, because some types of dysarthria exhibit loudness-related speech disturbances, such as reduced loudness, loudness decay, excess loudness variation, and monoloudness [3-6]. Particularly, the feature 'reduced loudness' could result from dysfunctions at various levels of speech production, such as insufficient respiratory support, inadequate phonatory function or nasal emission. Reduced loudness is assumed to have an effect on speech intelligibility, most simply because a louder signal should be more audible and better audibility seems, on logical analysis, to predict better speech in-

\section{KARGER \\ Fax +41613061234 \\ E-Mail karger@karger.ch}

() 2011 S. Karger AG, Basel

www.karger.com
Yunjung Kim, PhD

Department of Communication Sciences and Disorders

Louisiana State University

Baton Rouge, LA 70803 (USA)

Tel. +1 225578 3934, E-Mail ykim6@lsu.edu 
telligibility. Perhaps this explains why so much effort has been devoted to treatment of speech intensity (loudness production) in persons with dysarthria, and especially in persons with Parkinson's disease [6].

The history of treatment focusing on speakers' vocal effort for the purpose of speech rehabilitation goes back to Cooper et al. [7]. In this study, the authors suggested a multidisciplinary approach to managing parkinsonian speakers, where the speech therapy primarily emphasized 'boosting' techniques. Based on the findings, the authors stressed constant attention and self-awareness on the part of the patient to produce vocal intensity, to guarantee the desired results. This therapeutic approach has been systematically established in the Lee Silverman Voice Treatment (LSVT), which has been discussed as one of the most efficacious behavioral treatments for voice and speech disorders, primarily for speakers with Parkinson's disease [8]. The goal of LSVT is raising the habitual loudness of speech produced by individuals with dysarthria through a series of intense therapy sessions and home assignments. Previous literature has reported that the intensive practice of LSVT generates not only an increase in vocal loudness levels, but an enhancement of other speech aspects, including voice quality, articulatory integrity, speaking rate, and speech intelligibility [8-10]. As noted above, the gains in speech intelligibility are assumed at a first level of analysis to be related to increased audibility of sounds and words; improvement in articulatory function said to be associated with the increased vocal loudness level contributes to the assumption of improved audibility and speech intelligibility.

However, the relationship between vocal intensity and speech intelligibility is not straightforward. In other words, it is not yet understood how productive aspects of speech acoustic energy are related to speech intelligibility. A study by Tjaden and Wilding [11] reported a significant improvement of intelligibility scores scaled by freemodulus magnitude estimates for speakers with Parkinson's disease when their increased-level productions were compared to their habitual-level productions; a similar effect was not observed for speakers with multiple sclerosis. Even in the group with Parkinson's disease, the habitual-to-louder intelligibility improvement was not strongly related to changes in acoustic measures of articulation (such as size of the vowel space and lingual fricative distinctions). Moreover, in the recent literature on the effects of deep brain stimulation of the subthalamic nucleus as a palliative therapy for Parkinson's disease, evidence has been presented of therapy-induced dissociations between voice intensity and speech intelligibility. As reviewed by
Tripoliti and Limousin [12; see also 13, 14], some patients show increased voice intensity following deep brain stimulation, but decreased speech intelligibility. Similarly, Turner et al. [15] reported little change in word intelligibility and phonetic contrast errors across five presentation levels $(35,45,55,65,75 \mathrm{~dB} \mathrm{HL})$ for utterances produced by speakers with amyotrophic lateral sclerosis. Based on these findings, the authors supported the notion of a limited benefit of amplification for those speakers with word intelligibility deficits resulting from impairments in the speech production system beyond respiration $[16,17]$.

On the other hand, a recent study found significant increases in speech intelligibility (7-point scaling for sentence intelligibility and transcription for words) for both amplified and loud speech compared with speech produced at habitual levels by individuals with Parkinson's disease [18]. The author concluded that factors other than increased intensity must be partly responsible for the loud speech benefit because a greater intelligibility improvement was obtained from loud speech as compared to the amplification condition.

Because increases in voice SPL (and voice loudness) may involve covarying effects of or on articulation, it is useful to have a deeper understanding of how signal intensity affects estimates of speech intelligibility. Modifications of speech signal intensity, simply via amplification or attenuation, do not change articulation and therefore may permit an independent estimate of the relationship between signal intensity and intelligibility, as in the Neel [18] study. Moreover, it would be useful to have such data for scaled estimates of speech intelligibility, which are used frequently in studies of dysarthria [19, 20] and in popular clinical exams that may also be used in research studies (such as the UPDRS [21] and Frenchay $[22,23])$. The purpose of the present study was to explore the effects of the (1) presentation level and (2) across-sentence equalization of speech-signal intensities on direct magnitude estimates (DME) of speech intelligibility of sentences produced by persons with dysarthria and control speakers. By controlling the presentation level of speech signals ('high' vs. 'low'), we attempted to examine the effect of increased signal level reaching listeners' ears (in other words, eliminating possible by-products of intensity increase made by speakers, including improvement in articulation and vocal quality). By equalizing across-sentence intensities, we sought to determine how removing natural variation in vowel intensity (such as the 3- to 6-dB difference in a vowel such as /a/ as compared to $/ \mathrm{u} /$, even when both are produced with the same sub- 
Fig. 1. An example of filling the $\pm 6-\mathrm{dB}$ range with the most intense vocalic nucleus.

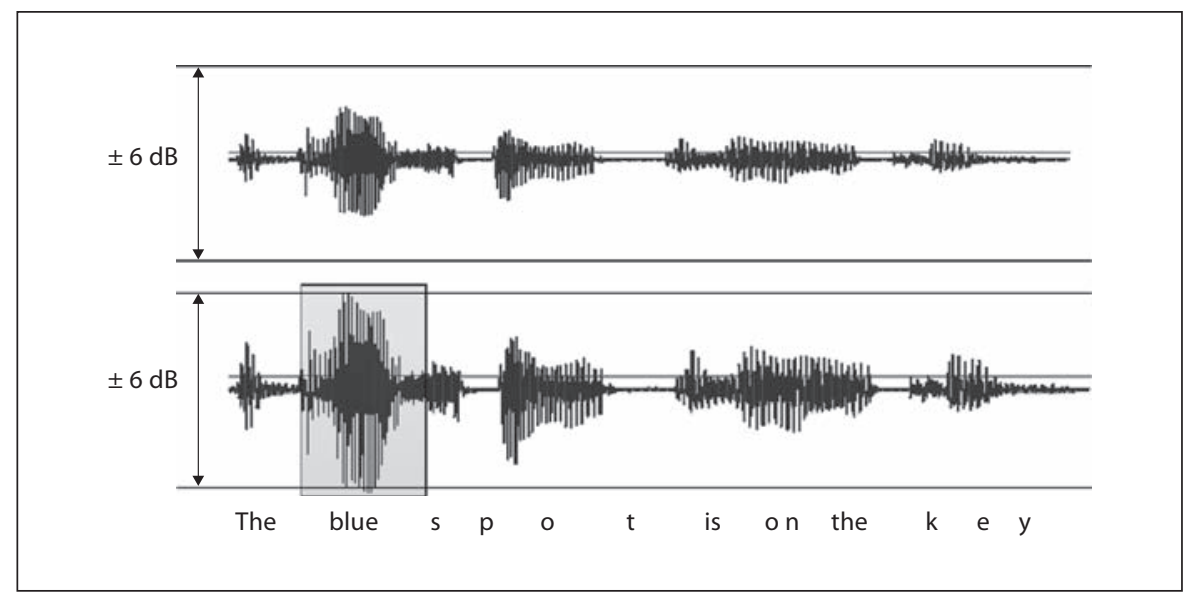

glottal pressure) might affect these estimates. Equalization of vowel intensities is not uncommon in studies of speech perception [24], but may disrupt normal intensity variation among vowels [25] and could potentially affect speech intelligibility.

\section{Methods}

\section{Speech Stimuli}

A total of 50 utterances was extracted from the archival database at the Speech Acoustics Laboratory, University of Wisconsin-Madison. These were originally recorded from 9 healthy speakers (21 utterances) and 16 speakers with dysarthria secondary to stroke, Parkinson's disease, and multiple system atrophy (29 utterances). The recordings were made in a quiet room with a high-quality microphone (SHURE SM 58) and a digital audio tape recorder (TASCAM DA-P1) at a sampling rate of $44.1 \mathrm{kHz}$ and with 16-bit quantization. Speakers with dysarthria were heterogeneous in etiology, speech severity and dysarthria type to encompass diverse phonatory and articulatory disturbances of dysarthria. All participants were asked to recite the following sentences in response to a demonstration by the examiner: (1) 'The blue spot is on the key', (2) 'The potato stew is in the pot', and (3) 'Combine all the ingredients in a large bowl'. Using these sentences, speech stimuli were prepared for experiments under four conditions: (1) 'high-adjusted', (2) 'high-unadjusted', (3) 'low-adjusted', and (4) 'low-unadjusted'; the nature of these conditions is described below.

Presentation levels were calibrated to the level of a $1,000-\mathrm{Hz}$ sinusoid, to which the frequent peaks of speech were matched as closely as possible. A sound level meter was placed $19 \mathrm{~cm}$ from a loudspeaker within a sound booth, the distance being chosen to match the distance of the listener's head from the loudspeaker. The 'high' presentation level was set at $80.5 \mathrm{~dB}$ SPL, the 'low' presentation level at $66.0 \mathrm{~dB}$ SPL. These levels were chosen for several reasons. First, we wanted to obtain DMEs at two levels that differed greatly in perceived loudness of the speech signal. Second, the 14.5-dB difference between the two levels was cho- sen because it approximated (1) the reported effect of LSVT on speech levels at least for vowel /a/ $[26,27]$ and (2) the amount of amplification provided by typical portable amplification systems for signals between 100 and $10 \mathrm{~Hz}$ [15]. Third, the levels were chosen, based on informal listening, to be somewhat louder and softer, respectively, than a 'normal' conversational voice lev$\mathrm{el}$ at this loudspeaker-to-listener distance. Sound level measurements with the sound booth door closed and no signal delivered through the speaker yielded an ambient measurement of $\sim 43 \mathrm{~dB}$ SPL.

The 'adjusted' condition equated the sentences for their most intense vocalic nucleus. For intensity adjustment, a 1,000-Hz calibration sinusoid was digitized and adjusted in relative intensity to fill a $\pm 6-\mathrm{dB}$ range, using the volume control in Sound Forge (Sony). Each sentence was then imported to Sound Forge and volume-adjusted until the most intense vocalic nucleus within the utterance filled the same \pm 6 - $\mathrm{dB}$ range. Because the adjustment was based only on the most intense vocalic nucleus in the original sentence, the other vocalic nuclei within the sentence did not reach the limits of the \pm 6 - $\mathrm{dB}$ range, but were scaled up or down with the target adjustment. The result of the collection of intensity adjustments was a set of fifty sentences equated for the level of their most intense vocalic nuclei. An example is shown in figure 1 .

The combination of two presentation levels ('high' vs. 'low') with either level-equalized sentences ('adjusted') or unequalized sentences ('unadjusted') yielded the four experimental conditions within which DME scale values were collected.

\section{Listening Task}

A total of 60 listeners were recruited for the study, who were college-aged adults with no hearing problems by self-report. Participants were assigned in series to one of the four conditions using a random number table. Each participant was seated in the sound booth and asked to scale speech intelligibility, using the DME technique. Speech intelligibility was defined as: 'How easy is it to understand exactly what the speaker said?' Listeners were told that higher numbers were to be used for more easily understood utterances, and lower numbers for less easily understood utterances. 


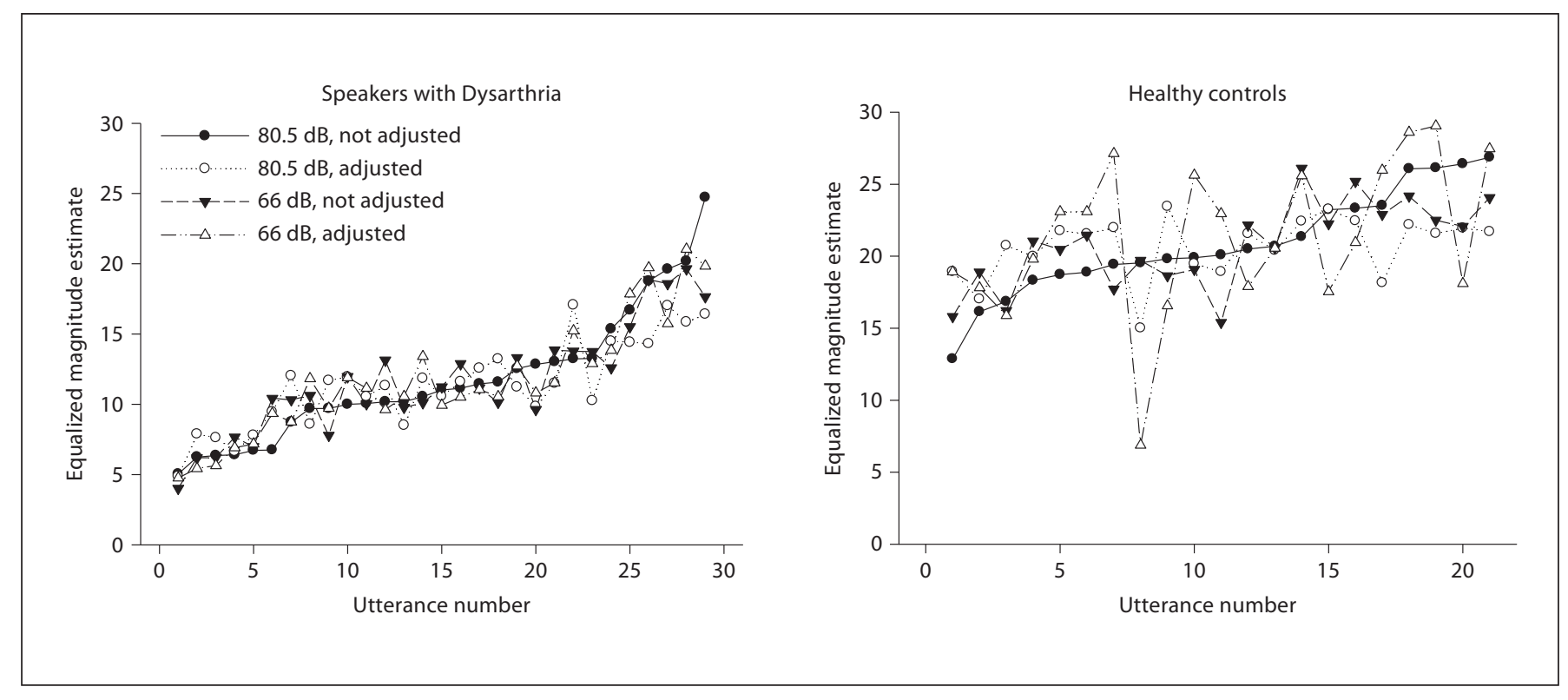

Fig. 2. Scaled speech intelligibility in order, from lowest to highest, using the 'high-unadjusted' condition as the reference; data for the other three conditions are plotted in the same rank order to show the covariation of scaled intelligibility across utterances. a Speakers with dysarthria. b Control speakers.

Experiments were conducted twice. In experiment 1, 40 listeners, 10 in each of the four conditions, were asked to scale speech intelligibility without a modulus to examine potential variations of speech intelligibility within each speaker group (dysarthria and control groups) as a function of level of presentation and level equalization. A modulus equalization procedure was followed to eliminate inter- and intra-observer variability caused by differing scale values used by different listeners [28]; this procedure transforms the across-listener scale values to a common scale.

Procedures were the same for the second experiment except that a fixed modulus was provided to listeners to permit direct comparisons across conditions and speaker groups. In experiment 2, 20 listeners participated (5 in each listening condition), none of whom had participated in the first experiment. Listeners were asked to make judgments relative to a modulus sentence assigned a value of 10 , which was chosen as a moderately dysarthric sample.

This study was reviewed and approved by the Human Subjects Committee of the Institutional Review Board at the University of Wisconsin-Madison. All participants signed an informed consent, consistent with the Declaration of Helsinki.

\section{Results}

In both experiments, $\mathrm{t}$ test results revealed that DME ratings were significantly lower for speakers with dysarthria compared to healthy controls $(\mathrm{p}<0.001)$, which indicates that speakers with dysarthria had speech intelligibility deficits. The differences in DME scale values be- tween health controls and speakers with dysarthria can be seen in figure 2 .

\section{Experiment 1: DME without Modulus}

When data are plotted by ranking utterances in one condition ('high-unadjusted') according to scaled intelligibility, from lowest to highest, and plotting all other conditions according to this ranking, the data appear as shown in figure 2 . Results showed that magnitude estimates ranged over a 4- to 5-fold factor among speakers with dysarthria, and roughly a 2.5 factor for normal speakers (fig. 2). When scale values were correlated across utterances for pairwise combinations of listening conditions, Pearson's correlation coefficients for speakers with dysarthria were very high and statistically significant for all combinations of conditions, and lower and statistically significant in only three combinations for control speakers (table 1).

These results suggest that among a set of utterances whose scaled speech intelligibility varies over a wide range, overall presentation level and equalization of peak intensity levels do not have much of an effect on the relative intelligibility of the utterances. This conclusion seems clear in the case of utterances produced by speakers with dysarthria and perhaps less convincing in control speakers. Based on this observation, the second ex- 
Table 1. Correlation coefficients between conditions for speakers with dysarthria and for healthy controls

\begin{tabular}{|c|c|c|c|c|c|c|}
\hline & \multicolumn{2}{|c|}{ Low-unadjusted } & \multicolumn{2}{|c|}{ Low-adjusted } & \multicolumn{2}{|c|}{ High-unadjusted } \\
\hline & DYS & $\mathrm{HC}$ & DYS & $\mathrm{HC}$ & DYS & $\mathrm{HC}$ \\
\hline Low-unadjusted & - & - & - & - & - & - \\
\hline Low-adjusted & $0.79^{*}$ & $0.69^{*}$ & - & - & - & - \\
\hline High-unadjusted & $0.86^{*}$ & NS & $0.87^{*}$ & NS & - & - \\
\hline High-adjusted & $0.82^{*}$ & NS & $0.89^{*}$ & $0.57^{*}$ & $0.91^{*}$ & $0.73^{*}$ \\
\hline
\end{tabular}

Significant level at ${ }^{*} \mathrm{p}<0.01$. DYS $=$ Dysarthria group HC $=$ healthy controls; NS = nonsignificant.

periment with fixed modulus was performed to augment the correlation analysis of the first experiment and make direct comparisons across listening conditions.

\section{Experiment 2: DME with Fixed Modulus}

The group data are presented in figure 3. Average speech intelligibility ratings were $15.19(\mathrm{SD}=5.29)$ for 'high-adjusted', 16.93 (SD = 6.53) for 'high-unadjusted', $13.92(\mathrm{SD}=6.41)$ for 'low-adjusted', and $16.04(\mathrm{SD}=6.15)$ for 'low-unadjusted' condition.

A two-way ANOVA was carried out to determine the influence of presentation level ('high' vs. 'low'), and level equalization adjustment of the vocalic nucleus ('adjusted' vs. 'unadjusted') on scaled speech intelligibility. The results showed the main effect of 'adjustment' to be significant $\left(\mathrm{F}_{1,19}=8.77 ; \mathrm{p}=0.003\right)$, while the main effect of the loudness level $\left(\mathrm{F}_{1,19}=2.72 ; \mathrm{p}=0.10\right)$ and the interaction between the loudness level and adjustment $\left(\mathrm{F}_{1,19}=0.083\right.$; $\mathrm{p}=0.77)$ were not significant.

Follow-up contrasts comparing intelligibility scores from intensity-adjusted and unadjusted utterances within loudness levels revealed that the unadjusted stimuli were scaled significantly more intelligible than adjusted stimuli (table 2).

\section{Reliability}

To obtain an estimate of interlistener reliability, an intraclass correlation coefficient was calculated for each condition in the two experiments [29]. Intraclass correlation coefficient ranged from 0.54 to 0.69 (experiment 1) and from 0.62 to 0.69 (experiment 2). Although the coefficients appear low especially compared to those obtained in the literature that utilized interval scaling for intelligibility measures, all intraclass correlation coefficients were statistically significant $(\mathrm{p}<0.000)$ and were judged to be satisfactory, considering that reliability is a major concern in using a DME technique. Intralistener reliabil-
Table 2. Follow-up contrasts between adjusted and unadjusted conditions within loudness levels

\begin{tabular}{llll}
\hline Conditions & Contrast & Mean difference & t value \\
\hline High & Adjusted-unadjusted & 1.74 & $3.28^{*}$ \\
Low & Adjusted-unadjusted & 2.12 & $3.77^{*}$ \\
\hline
\end{tabular}

Significant level at ${ }^{*} \mathrm{p}<0.01$.

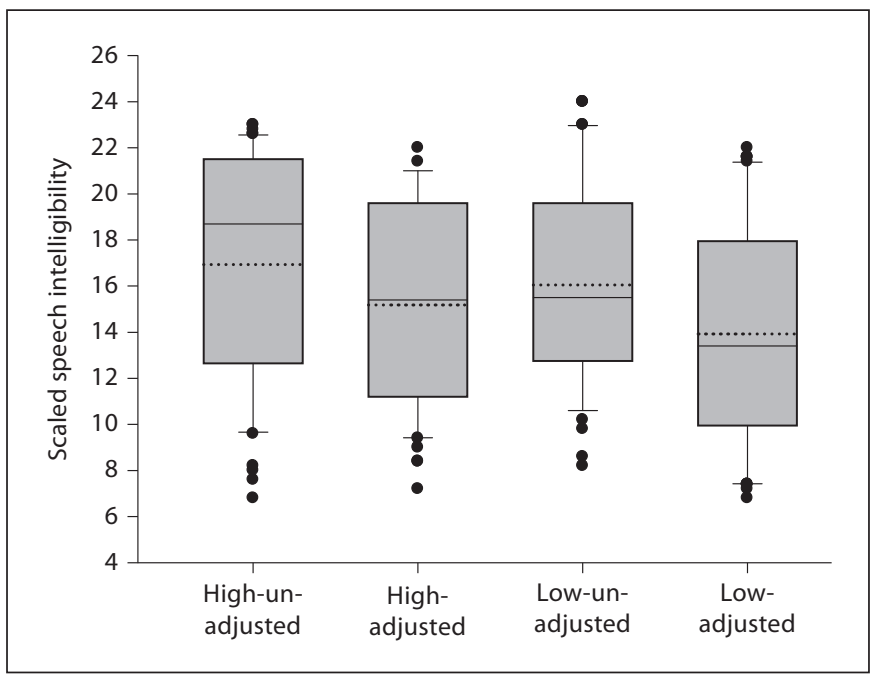

Fig. 3. Box and whisker plots of speech intelligibility for each condition. Mean (median) value is indicated by dotted (solid) lines.

ity is not reported. However, based on the significance of interlistener correlation coefficients, which are typically lower than intralistener correlation coefficients, interlistener correlation was regarded as a good estimate of listener reliability. 


\section{Discussion}

Partly due to the complexity of speech intelligibility, estimates of which are susceptible to a number of factors including speech stimuli, measuring procedures, and listeners' experience, it is challenging to specify the source of improvement following changes in certain behaviors of speakers. This study, in recognition of this issue, investigated the effect of presentation level on speech intelligibility of short sentences by using a DME technique with and without a modulus. The results suggest that an increase in the overall signal level alone does not guarantee significant improvement in speech intelligibility and that a change of the presentation level affects speech intelligibility ratings to a lesser degree for speakers with dysarthria compared to healthy controls. This finding is consistent with previously reported dissociations between speech signal intensity and speech intelligibility in patients with Parkinson's disease [12] and with a report of no effect of amplification on speech intelligibility of patients with amyotrophic lateral sclerosis [15]. On the other hand, some studies $[11,18]$ have shown improved intelligibility with increased voice intensity or amplified signals in speakers with Parkinson's disease. The different findings across the studies may have many explanations, but the conflicting findings make it clear that an increased speech signal level does not guarantee improved speech intelligibility.

These somewhat complicated results may stem from different experiment designs, subjects or speech intelligibility measures. First, it should be noted that speakers in this study were heterogeneous with respect to etiology, severity, and dysarthria type, and that most showed speech disturbances in more than intensity-related dimensions. This may be important in that it might have lessened the effect of the signal level on speech intelligibility in dysarthria compared to previous studies on Parkinson's disease, in which the primary concern is reduced intensity of speech (interestingly this was less emphasized in the original papers by Darley et al. $[3,4]$ compared to recent discussions that focus more on the overall reduction of loudness as a primary characteristic of this population, e.g. Fox et al. [6]). Some studies have pointed out that the greatest benefit of amplification occurs for individuals with only reduced vocal intensity [15-17]. Even speakers with the homogenous etiology of Parkinson's disease did not obtain the same increase in speech intelligibility from loud speech versus amplification [18]. Second, the current study had different blocks of listeners for each experimental condition in order to avoid the possi- bility where the 'relative' differences between experimental conditions are affected by listening to identical utterances across conditions. When listeners are assigned randomly to different conditions and do not repeat across conditions, the experiment should be most sensitive to true differences. The absence of a presentation level effect is therefore not due to a lack of sensitivity in the experimental design. In addition, the DME technique used in the study is not only sensitive to phonetic characteristics of utterances, but also to prosodic and voice quality characteristics that may have an effect on speech intelligibility [19].

Another interesting finding is that although the 'overall' increase in signal level did not affect speech intelligibility scaling, the equalization across utterances of the most intense vocalic nuclei caused significant decreases in speech intelligibility ratings, at least for the normal speakers. Across the utterances used in this experiment, the most intense vowel was sometimes a high vowel, sometimes a low vowel, depending on the speaker, the way in which a sentence was produced, and the position of the vowel in an utterance. It is therefore reasonable to assume that the equalization eliminated some of the natural vowel level differences in typical speech, and that this change might have degraded the naturalness of utterance and affected its intelligibility scale value.

Related to this, further investigation should evaluate the effect of stress patterns on speech intelligibility scaling. The current report did not include in the 'adjusted' condition any modification in stress pattern (more exactly in the relative magnitude of intensity contour across adjacent syllables), because the overall level was evenly changed for all syllables in the utterances. One thing that we do not know yet is how greater vocal effort affects the relative intensity across segments, syllables, and words, which, accordingly, is supposed to provide greater contrastivity of acoustic signals. This is interesting given that perceptual impressions such as monoloudness or monopitch were more frequently described as prominent perceptual features of dysarthria than overall reduced speech levels in Darley et al. [3, 4].

\section{Clinical Implications}

From the assumption that increased loudness will provide better audibility and empirical data from the LSVT and similar approaches that have reported positive treatment outcomes where the speaker's effort change at the laryngeal level spreads to effort throughout the speech mechanism, intensity management has been one of the frequently used behavioral approaches to management of 
speakers with dysarthria. Whereas the target population for voice intensity management was originally patients with Parkinson's disease, it has been extended to include individuals with stroke [30,31], traumatic brain injury [31,32], cerebral palsy [33], multiple sclerosis [34], and children with Down syndrome [35].

The present results of little or no improvement of scaled speech intelligibility following increasing presentation level lead to the possibility that only selected speakers with dysarthria (and other communication disorders as well) may be the best candidates for speech therapy techniques that involve an increase in vocal effort, unless the assumption is made that the voice effort change clearly affects articulatory gestures as well. The failure to demonstrate that intelligibility gains with increased voice level are accompanied by systematic articulatory changes [11] calls this assumption into question. The next question is what might be the standard for selecting good candidates for vocal-effort treatment, which is beyond the scope of the current study. However, the current results that a relatively large increase in the presentation level (especially compared to the 2.0- to 4.0-dB SPL voice level effect in connected speech samples in Parkinson's disease $[11,36])$ did not produce a significance change in speech intelligibility scale values, and that a change in the presentation level affected the scaling of the normal utterances to a greater degree (especially for midrange intelligibility) suggest that it should not automatically be assumed that voice effort therapy can be applied uniformly across patients. Rather more specific reasons must be determined for the basis of a speech intelligibility disorder to guide a therapy plan, such as the primary disturbance of the disorder, the cause of reduced volume, and/or speech severity.

\section{Acknowledgements}

The data reported in this paper were presented at the 157th and 158th meetings of the Acoustical Society of America. This study was supported by NIDCD DC00319.

\section{References}

1 Pollack I: Effects of high pass and low pass filtering on the intelligibility of speech in noise. J Acoust Soc Am 1948;20:259-266.

2 Kent RD, Wiley TL, Strennen ML: Consonant discrimination as a function of presentation level. Audiology 1979;18:212-224.

-3 Darley FL, Aronson AE, Brown JR: Differential diagnostic patterns of dysarthria. J Speech Hear Res 1969;12:246-269.

-4 Darley FL, Aronson AE, Brown JR: Clusters of deviant speech dimensions in the dysarthrias. J Speech Hear Res 1969;12:462-496.

5 Duffy J: Motor Speech Disorders: Substrates, Differential Diagnosis, and Management, ed 2. St Louis, Mosby, 2005.

-6 Fox CM, Ramig LO, Ciucci MR, Sapir S, McFarland DH, Farley BG: The science and practice of LSVT/LOUD: neural plasticityprincipled approach to treating individuals with Parkinson disease and other neurological conditions. Semin Speech Lang 2006;27: 283-299.

-7 Cooper IS, Riklan M, Stellar S, Waltz JM, Levita E, Ribera VA, Zimmerman J: A multidisciplinary investigation of neurosurgical rehabilitation in bilateral parkinsonism. J Am Geriatr Soc 1968;16:1177-1306.

-8 Spielman J, Ramig LO, Mahler L, Halpern A, Gavin WJ: Effects of an extended version of the Lee Silverman voice and speech in Parkinson's disease. Am J Speech Lang Pathol 2007;16:95-107.
-9 Baumgartner CA, Sapir S, Ramig LO: Voice quality changes following phonatory-respiratory effort treatment (LSVT) versus respiratory effort treatment for individuals with Parkinson disease. J Voice 2001;15:105-114.

10 Dromey C, Ramig LO, Johnson AB: Phonatory articulatory changes associated with increased vocal intensity in Parkinson disease: a case study. J Speech Hear Res 1995;38:751764.

11 Tjaden K, Wilding GE: Rate and loudness manipulations in dysarthria: acoustic and perceptual findings. J Speech Lang Hear Res 2004;47:766-783.

12 Tripoliti E, Limousin P: Electrical stimulation of deep brain structures and speech; in Massen B, van Lieshout P (eds): Speech Motor Control: New Developments in Basic and Applied Research. Oxford, Oxford University Press, 2010, pp 297-313.

13 Tripoliti E, Limousin P, Tisch S, Borrell E, Hariz MI: Speech in Parkinson's disease following subthalamic nucleus deep brain stimulation: preliminary results. J Med Speech Lang Pathol 2006;14:309-315.

-14 Tripoliti E, Zinzro L, Borrell E, Frost E, Tisch S, Martinez I, Hariz M, Limousin P: Effect of contact location and voltage amplitude on speech and movement in bilateral subthalamic nucleus deep brain stimulation. Mov Disord 2008;23:2377-2383.
15 Turner G, Martin H, de Jonge R: Influence of amplification on word intelligibility and phonetic contrast errors in dysarthria. J Med Speech Lang Pathol 2008;16:267-274.

16 Green MCL, Watson B: The value of speech amplification in Parkinson's disease patients. Folia Phoniatr Logop 1968;20:250-257.

17 Adams SG: Hypokinetic dysarthria; in McNeil MR (ed): Clinical Management of Sensorimotor Speech Disorders. New York, Thieme, 1997.

18 Neel AT: Effects of loud and amplified speech on sentence and word intelligibility in Parkinson disease. J Speech Lang Hear Res 2009; 52:1021-1033

19 Kim YJ, Kent RD, Weismer G: An acoustic study of the relationships among neurologic disease, dysarthria type, and severity of dysarthria. J Speech Lang Hear Res 2011;54:417429.

20 Weismer G, Laures JS: Direct magnitude estimates of speech intelligibility in dysarthria: effects of a chosen standard. J Speech Lang Hear Res 2002;45:421-433.

21 Fahn S, Elton RL, UPDRS Development Committee: Unified Parkinson Disease Rating Scale; in Fahn S, Marsden CD, Goldstein M, Calne DB (eds): Recent Developments in Parkinson's Disease. Florham Park, Macmillan, 1987, vol 2, pp 153-163.

$\checkmark 22$ Enderby P: Frenchay dysarthria assessment. Int J Lang Commun Disord 1980;15:165173. 
23 Enderby P, Palmer R: FDA2: Frenchay Dysarthria Assessment. Austin, Pro-Ed, 2008.

24 Miller SE, Schlauch RS, Watson PJ: The effects of fundamental frequency contour manipulations on speech intelligibility in background noise. J Acoust Soc Am 2010;128: 435-443.

25 Fant G, Kruckenberg A, Liljencrants J: The source-filter frame of prominence. Phonetica 2000;57:113-127.

-26 Ramig LO, Countryman S, Thompson LL, Horii Y: Comparison of two forms of intensive speech treatment for Parkinson disease. J Speech Hear Res 1995;38:1232-1251.

$\checkmark 27$ Ramig, LO, Sapir S, Countryman S, Pawlas AA, O’Brien C, Hoehn M, Thompson LL: Intensive voice treatment (LSVT) for patients with Parkinson's disease: a 2 year follow up. J Neurol Neurosurg Psychiatry 2001;71:493498.
28 Engen T: Psychophysics: discrimination and detection; in Kling JW, Riggs LA (eds) Woodworth and Schlossberg's Experimenta Psychology, ed 3. New York, Holt, 1971, pp 11-46.

29 Sheard C, Adams RD, Davis PJ: Reliability and agreement of ratings of ataxic dysarthric speech samples with varying intelligibility. J Speech Hear Res 1991;34:285-293.

30 Mahler LA, Ramig LO, Fox C: Intensive voice treatment (LSVT ${ }^{\circledR}$ LOUD) for dysarthria secondary to stroke. J Med Speech Lang Pathol 2009; 17/4.

31 Wenke RJ, Theodoros D, Cornwell P: The short- and long-term effectiveness of the LSVT for dysarthria following TBI and stroke. Brain Inj 2008;22:339-352.

32 Solomon NP, Makasha MJ, Kessler LS, Sullivan KW: Speech-breathing treatment and LSVT for a patient with hypokinetic-spastic dysarthria after TBI. J Med Speech Lang Pathol 2004;10:51-64.
33 Fox C, Boliek C, Ramig LO: The impact of intensive voice treatment (LSVT) on speech intelligibility in children with spastic cerebral palsy. Mov Disord 2005;20:S149.

>34 Sapir S, Pawlas A, Ramig LO, Seeley E, Fox C, Corboy J: Effects of intensive phonatoryrespiratory treatment $\left(\mathrm{LSVT}^{\circledR}\right)$ on voice in two individuals with multiple sclerosis. J Med Speech Lang Pathol 2001;9:141-151.

35 Audo CMC: Applying the Lee Silverman Voice Treatment (LSVT ${ }^{\circledR}$ ) to the Down Syndrome Population: A Single-Subject Design; MA thesis, University of Colorado, 2004.

36 Fox C, Ramig LO: Vocal sound pressure level and self-perception of speech and voice in men and women with idiopathic Parkinson disease. Am J Speech Lang Pathol 1997;6:8594. 